Reprinted from The Journal of Chemical Physics, Vol. 23, No. 4, 757-758, April, 1955

Printed in U. S. A.

\title{
Invariance of Integrated Transmittance and Total Fractional Transmission with Experimental Slit Function*
}

C. R. DePrima And S. S. Penner

California Institute of Technology, Pasadena, California (Received January 27, 1955)

$I^{1}$ $\mathrm{T}$ is well known to spectroscopists that the integrated transmittance and total fractional transmission are, in principle, independent of experimental slit width under the conditions usually satisfied in experimental work. A recent discussion of this problem for a triangular slit function has been given, for example, by Nielsen, Thornton, and Dale. ${ }^{1}$ It is the purpose of the present note to re-examine this question in more general terms and to specify the requirements under which the experimental results are, in principle, independent of the experimental slit function.

Consider the spectral region between the wave numbers $\omega_{1}$ and $\omega_{2}\left(\omega_{1}<\omega_{2}\right)$ which are selected in such a way that for $\omega \leqslant \omega_{1}$ $+\Delta \omega^{*}$ and for $\omega \geqslant \omega_{2}-\Delta \omega^{*}$ the spectral absorption coefficient $P(\omega)$ vanishes. The functional form of $P(\omega)$ in the range $\omega_{1}+\Delta \omega^{*}$ $<\omega<\omega_{2}-\Delta \omega^{*}$ is arbitrary. The spectral region from $\omega_{1}$ to $\omega_{2}$ is scanned with an instrument which can be described by a slit function $g\left(\left|\omega^{\prime}-\omega\right|, b, c\right)$ such that the instrument responds to the wave number $\omega^{\prime}$ when it is set at $\omega$. The slit function is assumed to vanish for $\left|\omega^{\prime}-\omega\right| \geqslant \Delta \omega^{*}$. The parameters $b$ and $c$ are dependent on the nature of the instrument selected for study. Representative slit functions are triangular, trapezoidal, or Gaussian. ${ }^{1}$

Let $I^{0}(\omega)$ represent the incident intensity, $I(\omega)$ is the transmitted intensity, $P(\omega)$ equals the spectral absorption coefficient, and $X$ is the optical density. The integrated transmittance is then

$$
\begin{aligned}
& \int_{\omega 1}^{\omega_{2}} T(\omega) d \omega=\int_{\omega 1}^{\omega_{2}} \\
& \quad \times\left(\frac{\int_{\omega-\Delta \omega^{*}}^{\omega+\Delta \omega^{*}} I^{0}\left(\omega^{\prime}\right)\left\{\exp \left[-P\left(\omega^{\prime}\right) X\right]\right\} g\left(\left|\omega^{\prime}-\omega\right|, b, c\right) d \omega^{\prime}}{\int_{\omega-\Delta \omega^{*}}^{\omega+\Delta \omega^{*}} I^{0}\left(\omega^{\prime}\right) g\left(\left|\omega^{\prime}-\omega\right|, b, c\right) d \omega^{\prime}}\right) d \omega .
\end{aligned}
$$

For spectrographs with reasonable resolution the intensity $I^{0}\left(\omega^{\prime}\right)$ may be taken as constant in the wave number range $\omega-\Delta \omega^{*} \leqslant \omega^{\prime}$ $\leqslant \omega+\Delta \omega^{*}$, i.e., $I^{0}(\tau+\omega)=I^{0}(\omega)$ for $|\tau| \leqslant \Delta \omega^{*}$. Hence, introducing an appropriate change of variables and changing the order of integration, we find

$$
\begin{gathered}
\int_{\omega 1}^{\omega_{2}} T(\omega) d \omega=\left[\int_{-\Delta \omega^{*}}^{\Delta \omega^{*}} g(|\tau|, b, c) d \tau\right]^{-1} \int_{\omega 1}^{\omega 2}\left(\int_{-\Delta \omega^{*}}^{\Delta \omega^{*}}\right. \\
\{\exp [-P(\tau+\omega) X] g(|\tau|, b, c) d \tau) d \omega=\left[\int_{-\Delta \omega^{*}}^{\Delta \omega^{*}} g(|\tau|, b, c) d \tau\right]^{-1} \\
\times \int_{-\Delta \omega^{*}}^{\Delta \omega^{*}} g(|\tau|, b, c)\left(\int_{\omega_{1}+\tau}^{\omega_{2}+\tau}\{\exp [-P(\omega) X]\} d \omega\right) d \tau .
\end{gathered}
$$

However, since $|\tau| \leqslant \Delta \omega^{*}$ the stated requirements for $P(\omega)$ imply that

$$
\int_{\omega_{1}+\tau}^{\omega_{2}+\tau}\{\exp [-P(\omega) X]\} d \omega=\int_{\omega_{1}}^{\omega_{2}}\{\exp [-P(\omega) X]\} d \omega .
$$

Consequently Eq. (2) reduces to

$$
\int_{\omega_{1}}^{\omega_{2}} T(\omega) d \omega=\int_{\omega_{1}}^{\omega_{2}}\{\exp [-P(\omega) X]\} d \omega .
$$

The experimental conditions which must be met in order to justify the use of Eq. (4) are seen to be the following: (a) if the slit function vanishes for $\left|\omega^{\prime}-\omega\right| \geqslant \Delta \omega^{*}$ and $P(\omega)$ vanishes for $\omega \leqslant \omega_{1}^{0}$ and $\omega \geqslant \omega_{2}^{0}$, we must scan at least over the spectral range from $\omega_{1}=\omega_{1}^{0}-\Delta \omega^{*}$ to $\omega_{2}=\omega_{2}^{0}+\Delta \omega^{* 3}$; (b) the incident intensity $I^{0}(\omega)$ must be a slowly varying function of $\omega$ such that $I^{0}(\omega+r)$ $\simeq I^{0}(\omega)$ for $|\tau| \leqslant \Delta \omega^{*}$.

The total fractional transmission of a spectral region $T_{R}$ is defined by the relation

$$
T_{R}=\frac{\int_{\omega_{1}}^{\omega_{2}}\left(\int_{\omega-\Delta \omega^{*}}^{\omega+\Delta \omega^{*}} I^{0}\left(\omega^{\prime}\right)\left\{\exp \left[-P\left(\omega^{\prime}\right) X\right]\right\} g\left(\left|\omega^{\prime}-\omega\right|, b, c\right) d \omega^{\prime}\right) d \omega}{\int_{\omega_{1}}^{\omega_{2}}\left(\int_{\omega-\Delta \omega^{*}}^{\omega+\Delta \omega^{*}} I^{0}\left(\omega^{\prime}\right) g\left(\left|\omega^{\prime}-\omega\right|, b, c\right) d \omega^{\prime}\right) d \omega}
$$

Proceeding as before, it is readily seen that Eq. (5) becomes

$$
T_{R}=\frac{\int_{-\Delta \omega^{*}}^{\Delta \omega^{*}} g(|\tau|, b, c) d \tau \int_{\omega_{1+\tau}}^{\omega_{2}+\tau} I^{0}(\omega)\{\exp [-P(\omega) X]\} d \omega}{\int_{-\Delta \omega^{*}}^{\Delta \omega^{*}} g(|\tau|, b, c) d \tau \int_{\omega 1+\tau}^{\omega_{2}+\tau} I^{0}(\omega) d \omega} .
$$

Hence, if $I^{0}(\omega)$ has the same values at corresponding points in the spectral ranges, $\omega_{1}-\Delta \omega^{*} \leqslant \omega \leqslant \omega_{1}+\Delta \omega^{*}$ and $\omega_{2}-\Delta \omega^{*} \leqslant \omega \leqslant \omega_{2}$ $+\Delta \omega^{*}$, respectively, then

$$
T_{R}=\frac{\int_{\omega_{1}}^{\omega_{2}} I^{0}(\omega)\{\exp [-P(\omega) X]\} d \omega}{\int_{\omega_{1}}^{\omega_{2}} I^{0}(\omega) d \omega}
$$

provided the previously stated conditions are met for the slit function and absorption coefficient. The conclusion that $I^{0}(\omega)$ is arbitrary, except in the neighborhood of the ends of the integration interval, is interesting and physically plausible.

* Supported by the Office of Naval Research under Contract Nonr220 (03), NR 015401.

Nielsen, Thornton, and Dale, Revs. Modern Phys. 16, 307 (1944).

${ }^{2}$ We must choose the integration interval larger by at least $\Delta \omega^{*}$ on either end than the range for which $P(\omega)$ is nonzero because, in principle, the spectrometer will show absorption when it is set within a range $\Delta \omega^{*}$ of the nearest absorbing region. 